\begin{tabular}{|c|l|}
\hline Title & Surface sliding friction of negatively charged polyelectrolyte gels. \\
\hline Author(s) & Kagata, Go; Gong, Jian Ping \\
\hline Citation & $\begin{array}{l}\text { Colloids and Surfaces B Biointerfaces, 56(1-2), 296-302 } \\
\text { https://doi.org/10.1016j.colsurfb.2006.10.034 }\end{array}$ \\
\hline Issue Date & 2007-04-15 \\
\hline Doc URL & http://hdl.handle.net/2115/26272 \\
\hline Type & article (author version) \\
\hline File Information & CSB56-1-2.pdf \\
\hline
\end{tabular}

Instructions for use 


\title{
Surface Sliding Friction of Negatively Charged Polyelectrolyte Gels
}

\author{
Go Kagata ${ }^{1}$ and Jian Ping Gong ${ }^{2,3, *}$ \\ ${ }^{1}$ Creative Research Initiative "SOUSEI”, Hokkaido University, Sapporo, 001-0021, Japan. \\ ${ }^{2}$ Department of Biological Science, Graduate School of Science, Hokkaido University, \\ Sapporo, 060-0810, Japan. \\ ${ }^{3}$ SORST, JST, Sapporo, 060-0810, Japan. \\ *To whom correspondence should be addressed. \\ E-mail: gong@sci.hokudai.ac.jp, Phone \& Fax: +81-11-706-2774
}

Keywords: polyelectrolyte gel, friction, hydrodynamic lubrication, interface interaction, electric-double layer

\begin{abstract}
.
The friction between two polyelectrolyte gels carrying the same or opposite sign of charges has been investigated using a rheometer. It is found that the friction was strongly dependent on the interfacial interaction between two gel surfaces. In the repulsive interaction case, especially, the friction was extremely low. The friction behavior is attempted to be described in terms of the hydrodynamic lubrication of the solvent layer between two like-charged gel surfaces, which is formed due to the electrostatic repulsion of the two gel surfaces. From the theoretical analysis (hydrodynamic mechanism), the friction behaviors were explained qualitatively, all of the experimental results, nevertheless, could not be understood well. The viscoelastic feature of the gel and the non-Newtonian behavior of water at the friction interface are considered to be important to elucidate the gel friction.
\end{abstract}

\section{Introduction.}

Some biological surfaces display fascinating low friction properties [1-10]. For example, cartilages of animal joints have a friction coefficient in the range 0.001-0.03, remarkably low even for hydrodynamically lubricated journal bearings [1,2]. It is not well understood why the cartilage friction of the joints is so low even in such conditions as that the pressure between the bone surfaces reaches as high as $3-18 \mathrm{MPa}$ and the sliding velocity is never greater than a few centimeters per second [1]. Under such conditions, the lubricating liquid layer cannot be sustained between two solid surfaces and the hydrodynamic lubrication does not work. Beside 
low surface friction, the articular cartilages, containing anionic proteoglycan-rich extracellular matrix (ECM), have remarkable elasticity and ability to withstand enormous physical forces[6,7]. These features are directly related to the high water content of cartilage, which is tightly held within the matrix of negatively charged macromolecular aggrecan/hyaluronic acid complexes stabilized by link proteins. Due to these facts, the negatively charged polyelectrolyte gels draw great attention despite considerable theoretical difficulties of the analysis.

Another example is the tribological role of polysaccharides on tissue and organ surfaces, such as mucus, which comprise a family of high molecular weight, extensively glycosylated glycoproteins. The mucus plays a crucial role in their biological activity, making smooth lubrication of tissues and organs and protecting cell surface from damage [8-10].

The authors consider that these fascinating tribological properties of the biological systems are originated from the soft and wet nature of tis sues and organs. That is, the role of solvated polymer network, especially the charged polymer network, existing in extracellular matrix as a gel state is critically important in the specific frictional behavior of the biological systems.

Studying on friction behavior of polyelectrolyte gels is helpful for understanding the low friction mechanism observed in biological systems, and may be useful in finding novel approaches in the design of low-friction artificial organs. This review intends to give a review of research on the surface sliding friction of negatively charged polyelectrolyte gels [11-28].

\section{Experiments.}

\section{2-1. Gel Preparation.}

2-Acrylamido-2-methylpropanesulfonic acid (AMPS), AMPS sodium salt (NaAMPS), quaternized $N$-[3-(dimethylamino) propyl] acrylamide (DMAPAA-Q), and acrylamido (AAm) were used as monomers for gel preparation. $N, N^{\prime}$-Methylenebisacrylamide (MBAA) used as a cross-lining agent was recrystallized from ethanol. Potassium persulfate used as a radical initiator was recrystallized from water.

Poly(2-Acrylamido-2-methylpropanesulfonic acid) (PAMPS) and its sodium salt (PNaAMPS) gels were prepared by radical polymerization of a $1 \mathrm{~mol} / \mathrm{L}$ aqueous AMPS (or NaAMPS) solution in the presence of MBAA and potassium persulfate. The polymerization was carried out at $60^{\circ} \mathrm{C}$ for $12 \mathrm{~h}$ under $\mathrm{N}_{2}$ atmosphere. PAMPS gels with different water content were prepared by changing the amount of MBAA.

Poly(quaternized $N$-[3-(dimethylamino)propyl] acrylamide) (PDMAPAA-Q) gel were prepared with the same method. 
Copolymer gels of AAm and NaAMPS (P(AAm-co-NaAMPS)) with different compositions were prepared by radical polymerization of NaAMPS with AAm in water, by varying the mole fraction of AAm, in the presence of MBAA. Copolymer gels of AAm and DMAPAA-Q (P(AAm-co-DMAPAA-Q)) with different compositions were prepared in the same way as that of P(AAm-co-NaAMPS) gel.

All of the above gels were prepared between two parallel glass plates to give sheet-shaped gels. After polymerization, the gels were immersed in a pure water to equilibrate swelling. The water content in gels was characterized by the swelling degree, $q$, calculated as the weight ratio of swollen gel to dry gel.

\section{2-2. Measurements.}

The friction force of gels was measured in water using a rotary rheometer (ARES, TA Instruments Inc.). The as-prepared gels were cut to form disks $15 \mathrm{~mm}$ in diameter and these were fixed on the upper surface of coaxial disk-shaped platen. Another piece of a gel was fixed on the lower platen as the friction substrate. The interface was immersed in water. The friction force was measured as the torque when the lower platen rotated with an angular velocity, $\omega$. The details of the measurement are described in our previous papers $[14,17,20]$

\section{Experimental Results.}

The effect of interfacial interaction on the gel friction can be observed simply and clearly from the friction behavior between two gels carrying charges. When two polyelectrolyte gels having opposite charges, for example, PAMPS gel and PDMAPAA-Q gel, were attempted to slide over each other, the adhesion between two gel surfaces was so high that both gels were broken. This strong adhesion should be apparently attributed to the electrostatic attraction between the polyanion ic (PAMPS) and the polycationic (PDMAPAA-Q).

On the other hand, the friction between like-charged gels was extremely low. Fig.1 shows the network charge density dependence of the friction between two PAMPS gels. Charge density modulation of PAMPS gel was made by varying the amount of cross-linking agent in the gel preparation, which gave different swelling ability to gels. Since the polyelectrolyte gels used in this study dissociate fully in water, carrying one charge for each monomer unit, the charge density $c[\mathrm{~mol} / \mathrm{L}]$ equals the concentration of monomer unit. So, $c=1000 / q M_{\mathrm{w}}$ $\left(M_{\mathrm{w}}\right.$ : molecular weight of the monomer). As shown in Fig.1, the friction increases modestly with increasing in charge density. That is, the friction force does not change sensitively with the swelling degree $q$. In this case, two opposite effects can be considered by examining the 
increase in charge density: One is the enhanced electrostatic repulsion between two gels, which favors the formation of a thicker water layer between the surfaces and decreases the friction. The other is the increased network density, which increases the hydrodynamic resistance of the water in the network and leads to an increase in friction force. Higher water content would lead to a small friction since the fraction of polymer network that is non-flow decreases with increasing in the water content. Therefore, a higher charge density would bring a thicker water layer, which leads to a lower friction but gives rise to an increase of non-flow network density, which increases the friction. These two opposite effects on the friction explain why the friction is not sensitive to the network charge density.

The effect of charge density was also studied by sliding homopolymer gels against copolymer gels containing different numbers of ionic monomers. Fig.2a shows the friction coefficient when $\mathrm{P}(\mathrm{AAm}-\mathrm{co}$-NaAMPS) gels with various composition were slid on a PNaAMPS gel. With the increase in AAm composition, that is, decrease in the negative charge density, the friction increased clearly. Similar results were obtained when the P(AAm-co-PDMAPAA-Q) gel were slid against PDMAPAA-Q gel. Taking into account the change in the swelling degree (Fig.2b), the charge density of copolymer gels was calculated. The relation between the charge density of copolymer gels and the friction coefficient is shown in Fig.3. The friction decreases very clearly with the increase in the charge density. In this regard, however, we cannot explain well why P(AAm-co-PDMAPAA-Q) gel on PDMAPAA-Q gel showed a higher friction than the P(AAm-co-NaAMPS) gel on PNaAMPS gel. The interaction between AAm and DMAPAA-Q might be stronger than that between AAm and NaAMPS.

As the further study, we attempted to investigate the effects of the friction velocity on the gel friction if the two gels slid on each other were likely-charged. Fig.4a shows the angular velocity dependence of the friction between two pieces of PNaAMPS gels in water at $20^{\circ} \mathrm{C}$. This test was performed under various normal pressures. At small pressure $(2 \mathrm{kPa})$, the friction force shows no dependence on the velocity over a range of 3 orders in magnitude. At larger pressures (higher than $5 \mathrm{kPa}$ ), the friction force, $f$, begins to show stronger dependence on the velocity, $\omega$. For simplicity, a first order fit was used to approximate data, i.e., $f \propto \omega^{\beta}$. The exponent $\beta$ increases with the increase in the normal pressure and saturates to a value about 0.55 at a high pressure, as shown in Fig.4b. This result will be discussed in the next section. 


\section{Theoretical Analys is.}

According to our repulsive interaction model for the gel friction $[12,14]$, the low friction between like-charged gels is attributed to the hydrodynamic mechanism, in which the strong electrostatic repulsion plays an important role in forming a solvent layer at the interface. Here, the thickness of the solvent layer at the interface will be estimated. When the applied normal pressure is not very high, the electrostatic repulsion prevails and the van der Waals interaction between two networks can be neglected. Supposing that the polyions having one charge for each monomer unit are distributed homogeneously in the bulk gel as well as on the gel surface, the average surface charge density $\sigma\left[\mathrm{m}^{-2}\right]$ can be given as

$$
\sigma=\left(10^{3} c N_{\mathrm{A}}\right)^{2 / 3}=\left(10^{6} N_{\mathrm{A}} / q M_{\mathrm{w}}\right)^{2 / 3},
$$

where, $c[\mathrm{~mol} / \mathrm{L}]$ is the charge density of the bulk gel and $N_{\mathrm{A}}$ is the Avogadro's number.

The solvent layer thickness at the interface is denoted as $2 l$ and the gel thickness as $L$ ( > $l$ ), and the $x-z$ coordinate system is set (Fig.5).

The electrical potential distribution between two gel surfaces, $\psi(z)$, given by Poisson-Boltzmann (PB) equation is expressed as

$$
\frac{\mathrm{d}^{2} \psi}{\mathrm{d} z^{2}}=-\frac{e n_{0}}{\varepsilon} \exp (-e \psi / k T), \quad-l<z<l
$$

where $\varepsilon$ is the dielectric constant of solvent (water), $e$ the elementary electron charge, $n_{0}$ the density of counterions at $z=0$ where $\psi(0)=0, k$ the Boltzmann constant, and $T$ is the absolute temperature. For simplicity, the case of monovalent ions is only considered. On the gel surface,

$$
\left.\frac{\mathrm{d} \psi}{\mathrm{d} z}\right|_{z= \pm l}=\mp \frac{e \sigma}{\varepsilon} .
$$

On the symmetric plane of $z=0$,

$$
\left.\frac{\mathrm{d} \psi}{\mathrm{d} z}\right|_{z=0}=0
$$

From the electrical neutrality,

$$
\sigma=n_{0} \int_{0}^{l} \exp (-e \psi / k T) \mathrm{d} z
$$

By choosing $\psi_{z=0}=0$, Eq.(2) can be solved analytically,

$$
\frac{e \psi}{k T}=2 \ln \cos \left(z \sqrt{\frac{n_{0} r_{0}}{2}}\right),
$$

where $n_{0}$ is a function of the surface number charge den sity given by 


$$
\sigma=\sqrt{\frac{2 n_{0}}{r_{0}}} \tan \left(l \sqrt{\frac{n_{0} r_{0}}{2}}\right) .
$$

Here, $r_{0}=e^{2} / \varepsilon k T$ is a constant with a dimension of length.

The repulsive osmotic pressure $\Pi$ between two approaching charged surfaces is determined by the microion charge density at the symmetric plane of $z=0$, where the electrostatic attraction on the microions is zero since $\mathrm{d} \psi /\left.\mathrm{d} z\right|_{z=0}=0$, so

$$
\Pi=n_{0} k T \text {. }
$$

In the equilibrium state, $\Pi$ is counterbalanced by the applied normal pressure $P$. Thus, by combining Eq.(7) and (8), the solvent layer thickness $2 l$ under a pressure $P$ is obtained as,

$$
2 l=2 \sqrt{\frac{2 k T}{P r_{0}}} \arctan \left(\sigma \sqrt{\frac{k T r_{0}}{2 P}}\right) .
$$

When $\sigma$ is very small and $P$ is high so that $\sigma \sqrt{k T r_{0} / 2 P}<<1$ is satisfied, $\arctan \left(\sigma \sqrt{k T r_{0} / 2 P}\right) \cong \sigma \sqrt{k T r_{0} / 2 P}$. Accordingly, $2 l \propto P^{-1}$. On the other hand, when $\sigma$ is very large and $P$ is not too high so that $\sigma \sqrt{k T r_{0} / 2 P}>>1, \arctan \left(\sigma \sqrt{k T r_{0} / 2 P}\right) \cong \pi / 2$. Accordingly, $2 l \propto P^{-1 / 2}$. This indicates that the highly charged surfaces are able to sustain more pressure.

Fig.6 shows the theoretical relations between $2 l$ and $P$ at various swelling degree of the gel. At high surface charge densities, that is, at low swelling degrees, the repulsion distance $2 l$ is not sensitive to the pressure $P$ when $P$ is not very high. Notice that the experimental investigation in Fig.1 was made at $q=10-30$, and $P \approx 10^{4} \mathrm{~Pa}$. Under these conditions, the solvent layer thickness $2 l$ is not sensitive to the surface charge density and it has a value of ca. $30 \mathrm{~nm}$.

When the two gel surfaces intermediated by a water layer of thickness $2 l$ are allowed to slide each other, the shear flow of the water layer exerts shear forces on the gel surfaces and gives rise to friction. Here, it cannot be considered that a non-slippery boundary is located at the gel surface because the gel consists of an elastic polymer network and viscous water; the latter can flow under the shear stress. That is, the velocity profiles in the gel regions should be taken into consideration. The flow of solvent in a polymer network is expressed successfully by the Debye-Brinkman equation, in which the effect of the polymer network is represented by a distributed shear force $-\eta v / K_{\text {gel }}$ [29-31]. Here, $\eta$ and $v$ are the viscosity and flow 
velocity of solvent in the network, respectively. $K_{\text {gel }}$ is the permeability of the gel. Using the Debye-Brinkman model, the shear stress, $f$, at the gel surface can be expressed as

$$
f=-\eta\left(\frac{\mathrm{d} v}{\mathrm{~d} z}\right)_{z= \pm l}=\frac{\eta v_{0}}{2\left(l+\sqrt{K_{\text {gel }}}\right)},
$$

where, $v_{0}$ is the relative velocity between the upper and the lower gels (Fig.5). Eq.(10) indicates that the shear flow can penetrate in to the gel with a thickness of $\sqrt{K_{\text {gel }}}$, or the equivalent non-slippery boundary is located at a depth of $\sqrt{K_{\text {gel }}}$ from the gel surface.

Eq.(10) also indicates the friction is in inversely proportion to $l+\sqrt{K_{\text {gel }}}$. With the increase in the network density, $\sqrt{K_{\text {gel }}} \cong \xi$ decreases but on the other hand, $l$ increases. So, the dependence of $\xi / l$ on the swelling degree $q$ as well as on the pressure is critically important in determining the friction force. When $q$ is not too large, $\xi / l<<1$ and the effect of $\xi$ can be neglected, but for very large $q, \xi$ becomes significant and should be taken into consideration.

Next, the case of two gels rotating with relative angular velocity $\omega_{0}$ is considered. For a liquid existing between two infinitely large coaxial plates rotating, its spatial velocity profile is very complicated [32,33]. But for the present case, the Reynolds number of motion, $\operatorname{Re} \approx \omega_{0} l^{2} / v<<1$, so only the angular velocity component is predominant [32]. The shear stress on the gel surfaces at a distance $r$ from the rotation axis is

$$
f=-\eta\left(\frac{\mathrm{d}}{\mathrm{d} z} r \omega\right)_{z= \pm l}=\frac{\eta r \omega_{0}}{2\left(l+\sqrt{K_{\mathrm{gel}}}\right)} .
$$

So, the total friction force $F$ for a disk-shaped gel of radius $R$ is

$$
F=\int_{0}^{R} 2 \pi r \cdot f \mathrm{~d} r=\frac{\pi \eta \omega_{0} R^{3}}{3\left(l+\sqrt{K_{\text {gel }}}\right)} .
$$

From the above equation, the friction force per unit area, $f$, is proportional to the angular velocity and the radius of the gel, since the average velocity increases with the radius. Fig.7 demonstrates the dependence of the friction coefficient, which is calculated from $\mu=F / \pi R^{2} P$, on the swelling degree for two ionized gels of the same kind undergone relative rotation at a certain applied normal pressure $P$. At a wide range of $P$, the friction is not sensitively dependent on the swelling degree. This is due to the cancellation of the opposite effects of the swelling degree on the solvent layer thickness $2 l$ and on the gel 
permeability $\sqrt{K_{\text {gel }}}$, as was discussed previously. This result explains well why the observed friction coefficient in Fig.1 did not depend sensitiv ely on the degree of swelling.

Under the experimental condition: $q=10-30$ and $P \approx 10^{4} \mathrm{~Pa}$, Fig.7 gives a friction coefficient $\mu \approx 10^{-3}$. This theoretically calculated value is about 1 order of magnitude lower than that of the experimental in Fig.1. This discrepancy may be associated with the so-called "bound water". As has been verified experimentally, the thermal movement of water molecules located adjacent to the macro-ions is locally restricted and ${ }^{1} \mathrm{H}$ NMR spin-lattice relaxation time [34] is low due to strong attractive interaction with macro-charges located nearby. This suggests that the water molecules, which are located within a certain distance from the macro-ions, move out hardly from the potential energy valley made by the charged network. On the other hand, free water existing far from the ionic atmosphere can migrate easily under external stimuli such as an electric field, hydrostatic pressure, or, in the present case, the shear force. From the research on the electric field induced contraction of PAMPS gels [35], which occurs due to the electro-osmotic migration of water in the gel, about 148 water molecules per sulfonate moiety are bound to the polymer chain strongly and not displaced by the electric field. This corresponds to water molecules absorbed around the macro-ions at a thickness of 3-4 times the radius of the macro-ions. In addition, it was also showed experimentally that the electro-osmotic migration velocity of water in the PAMPS gel is only $1 / 10$ of that from theoretical estimation, in which the free water viscosity had been used. Therefore, using the bulk viscosity of water would underestimate largely the friction force.

From the above theoretical analysis, the gel friction in the repulsive interaction case could be explained qualitatively. However, as shown in Fig.4, the velocity dependence of the friction does not show good agreement with the theoretical prediction. Although the friction force increases monotonously with an increase in angular velocity, the relationship between $f$ and $\omega$ is far more complicated than what can be expected from the simple hydrodynamic mechanism, which predicts $\beta=1$ (Eq.(11)). Especially, we cannot explain well why the frictional force does not depend on the sliding velocity when the normal pressure is low. Two possible factors, (i) the shear thinning effect of a gel and (ii) the non-Newtonian behavior of water at the friction interface, might be important.

(i) The highly hydrated polymer network of a gel might be extensively deformed under the shear stress (friction force) at the interface due to its viscoelastic nature. Therefore, the larger the sliding velocity, the higher is the shear stress and the more the network is deformed. This, 
in turn, would result in an increased interface gap between two gel surfaces, which leads to a decrease in the frictional force. Nevertheless, this tentative explanation is considered to be not sufficient because of the fact that the friction force is constant over 3 orders in magnitude of rotational velocity.

(ii) If water molecules form a layer at the friction interface and behave ideally as a Newtonian fluid, the static friction should not be observed at the beginning of interface shearing. However, our studies show that the interface between two repulsive gel surfaces could not slip with each other until the shear stress acting on the interface exceeded over a critical value. Solid marks in Fig.8 shows the time profile of the friction shear stress when two PNaAMPS gels are rotated relatively at an angular velocity of $10^{-2} \mathrm{rad} / \mathrm{s}$ in water. Open marks, on the other hand, shows the torsional shear stresses when two gel surfaces were totally glued together, and the shear was applied in a similar manner. In this case, the detected torque is due to a torsional deformation of the gels behaving as one continuous body. As shown in Fig.8, the frictional and torsional shear stresses overlap exactly with each other until the friction stress reached a peak value. This result informs us of an indisputable fact that the two like-charged gel surfaces, which are compressed under a normal pressure, cannot slip with each other until the shear stress acting on the interface exceeds a certain critical value, as if the two surfaces are glued together. This result suggests that the water molecules, which are hydrated strongly to the polyions and forms a layer at the interface, possess extremely high viscosity or even behave like a non-Newtonian fluid under the constraint interface environment.

The above results demonstrate that the mechanism of gel friction in the repulsive case cannot be explained simply in terms of hydrodynamic lubrication. Probably, two effects (the shear thinning effect of polymer networks and non-Newtonian behavior of water molecules) intertwined intricately with each other make a vitally important contribution to friction. The explanations given for the trends in the repulsive case are not conclusive and further studies are needed.

\section{Conclusions.}

From the above experimental results, it is demonstrated that the gel friction is largely dependent on the electrostatic interaction between two gel surfaces. The low friction between two like-charged gels must be mainly under the domination of the hydrodynamic nature of the interface. However, all of the experimental results cannot explain by the theoretical analysis, which suggests the simple hydrodynamic mechanism is insufficient to represent the friction 
behaviors comprehensively. The viscoelastic feature of the gel and the non-Newtonian behavior of water at the interface must be considered in order to elucidate the friction mechanism.

\section{References.}

1. C.W. McCutchen, Wear, 5 (1962) 1.

2. C.W. McCutchen, Lubrication of joints: the joints and synovial fluid, Academic Press, New York, 1978.

3. D. Dowson, A. Unsworth and V. Wright, J. Mech. Eng. Sci., 12 (1970) 364.

4. G.A. Ateshian, H.Q. Wang and W.M. Lai, J. Tribol., 120 (1998) 241.

5. W.A. Hodge, R.S. Fijian, K.L. Carlson, R.G. Burgess, W.H. Harris and R.W. Mann, Proc. Natl. Acad. Sci. USA, 83 (1986) 2879.

6. A.J. Grodzinsky, CRC Crit. Rev. Biomed. Eng., 9 (1985) 133.

7. M.D. Buschmann and A.J. Grodzinsky, J. Biomech. Engng., 117 (1995) 179.

8. E.M. Wojtys and D.B. Chan, Instr. Course Lect., 54 (2005) 323.

9. B.N.J. Persson, Sliding Friction: Physical Principles and Applications, 2nd ed., NanoScience and Technology Series, Springer, Berlin, 1998.

10. Y.C. Fung, Biomechanics: Mechanical properties of living tissues, 2nd ed., Springer-Verlag New York Inc., 1993.

11. J.P. Gong, M. Higa, Y. Iwasaki, Y. Katsuyama and Y. Osada, J. Phys. Chem. B, 101 (1997) 5487.

12. J.P. Gong and Y. Osada, J. Chem. Phys., 109 (1998) 8062.

13. J.P. Gong, Y. Iwasaki, Y. Osada, K. Kurihara and Y. Hamai, J. Phys. Chem. B, 103 (1999) 6001.

14. J.P. Gong, G. Kagata and Y. Osada, J. Phys. Chem. B, 103 (1999) 6007.

15. J.P. Gong, Y. Iwasaki and Y. Osada, J. Phys. Chem. B, 104 (2000) 3423.

16. J.P. Gong, T. Kurokawa, T. Narita, G. Kagata, Y. Osada, G. Nishimura and M. Kinjo, J. Am. Chem. Soc., 123 (2001) 5582.

17. G. Kagata, J.P. Gong and Y. Osada, J. Phys. Chem. B, 106 (2002) 4596.

18. T. Kurokawa, J.P. Gong and Y. Osada, Macromolecules, 35 (2002) 8161.

19. T. Baumberger, C. Caroli and O. Ronsin, Phys. Rev. Lett., 88 (2002) 75509.

20. G. Kagata, J.P. Gong and Y. Osada, J. Phys. Chem. B, 107 (2003) 10221.

21. T. Baumberger, C. Caroli and O. Ronsin, Eur. Phys. J. E., 11 (2003) 85.

22. Y. Ohsedo, R. Takashina, J.P. Gong and Y. Osada, Langmiur, 20 (2004) 6549. 
23. T. Tada, D. Kaneko, J.P. Gong, T. Kaneko and Y. Osada, Tribology Letters, 17 (2004) 505.

24. D. Kaneko, T. Tada, T. Kurokawa, J.P. Gong and Y. Osada, Advanced Materials, 17 (2004) 535.

25. T. Kurokawa, T. Tominaga, Y. Katsuyama, R. Kuwabara, H. Furukawa, Y. Osada and J.P. Gong, Langmiur, 21 (2005) 8643.

26. Y. Nitta, H. Haga and K. Kawabata, J. Phys. IV France, 12 (2002) 319.

27. A. Kii, J. Xu, J.P. Gong, Y. Osada and X.M. Zhang, J. Phys. Chem. B, 105 (2001) 4565.

28. T. Narita, A. Knaebel, J.P. Munch, S.J. Candau, J.P. Gong and Y. Osada, Macromolecules, 34 (2001) 5725.

29. H.C. Brinkman, Physica, 13 (1947) 447.

30. F.W. Wiegel, Lecture Notes in Phys., Springer, Berlin, 1980.

31. C.R. Ethier and R.D. Kamm, PCH Physico Chem Hydrodyn., 11 (1989) 219.

32. G.L. Mellor, P.J. Chapple and V.K. Stokes, J. Fluid Mech., 31 (1968) 95.

33. S.M. Roberts and J.S. Shipman, J. Fluid Mech., 73 (1976) 53.

34. H. Yasunaga and I. Ando, Polym. Gels Networks, 1 (1993) 83.

35. J.P. Gong, T. Nitta and Y. Osada, J. Phys. Chem., 98 (1994) 9583. 


\section{Figure Captions.}

Figure 1 [14]: Charge density and swelling degree dependencies of friction coefficient between two PAMPS gels in water. Angular velocity $\omega=0.05 \mathrm{rad} / \mathrm{s}$. Initial normal force $W=3 \mathrm{~N}$.

Figure 2 [14]: Dependencies of friction coefficients (solid marks) and swelling degree (open marks) on the copolymer composition. The frictions are the values between P(AAm-co-NaAMPS) gel and PNaAMPS gel $(\bullet, \circ)$, or P(AAm-co-DMAPAA-Q) gel and PDMAPAA-Q gel $(\square, \square)$ in water. Angular velocity $\omega=0.01 \mathrm{rad} / \mathrm{s}$. Initial normal force $W=3 \mathrm{~N}$.

Figure 3 [14]: Relation between the friction coefficient and the copolymer gel charge density calculated from Fig.2. (•) P(AAm-co-NaAMPS); ( $\Delta$ ) P(AAm-co-DMAPAA-Q).

Figure 4 [17]: (a) Angular velocity dependence of the friction force for a PNaAMPS gel rotated on a piece of PNaAMPS gel in water. The numbers in the figures are the normal pressures. (b) Relationships between the normal pressure and the scaling exponent $\beta$ of $f \propto \omega^{\beta}$ for PNaAMPS gel.

Figure 5: The geometry of two like-charged gels approaching with each other in water. Lower gel is made a motion in a velocity $v_{0}$.

Figure 6 [14]: Relationship between the water layer thickness $2 l$ and the normal pressure for charged gels with various swelling degree $q$. Following the dotted arrow direction in the figure, $q=1,10^{2}, 10^{3}, 10^{4}$. Parameters used in the calculation: $\varepsilon=78 \varepsilon_{0}, T=300 \mathrm{~K}$, and $M_{\mathrm{W}}=229$ (of NaAMPS).

Figure 7 [14]: Dependencies of the friction coefficients on the swelling degree and on the charge density for two charged gels undergone relative rotation. Numbers in the figure are the normal pressures in $\mathrm{Pa}$. Parameters used in the calculation: $\omega_{0}=0.05 \mathrm{rad} / \mathrm{s}, R=7.5 \mathrm{~mm}$, and $\eta=10^{-3}$ Pas (of bulk water). 
Figure 8 [20]: Frictional $(\bullet)$ and torsional (०) shear stresses as a function of shearing time measured in water at $20^{\circ} \mathrm{C}$. Normal pressure: $14 \mathrm{kPa}$. An gular velocity: $10^{-2} \mathrm{rad} / \mathrm{s}$. 
Manuscript (including Figs)
Click here to download Supplementary Material: Manuscript(includingFigs).pdf

Manuscript (including Figs)
Click here to download Supplementary Material: Manuscript(includingFigs).pdf

to

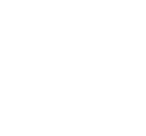

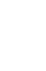

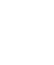

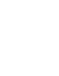
.

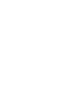

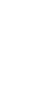


更 更 更 更 更 更 更 更

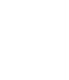
更 更 更 更 更 更 
Figure 1
Click here to download high resolution image

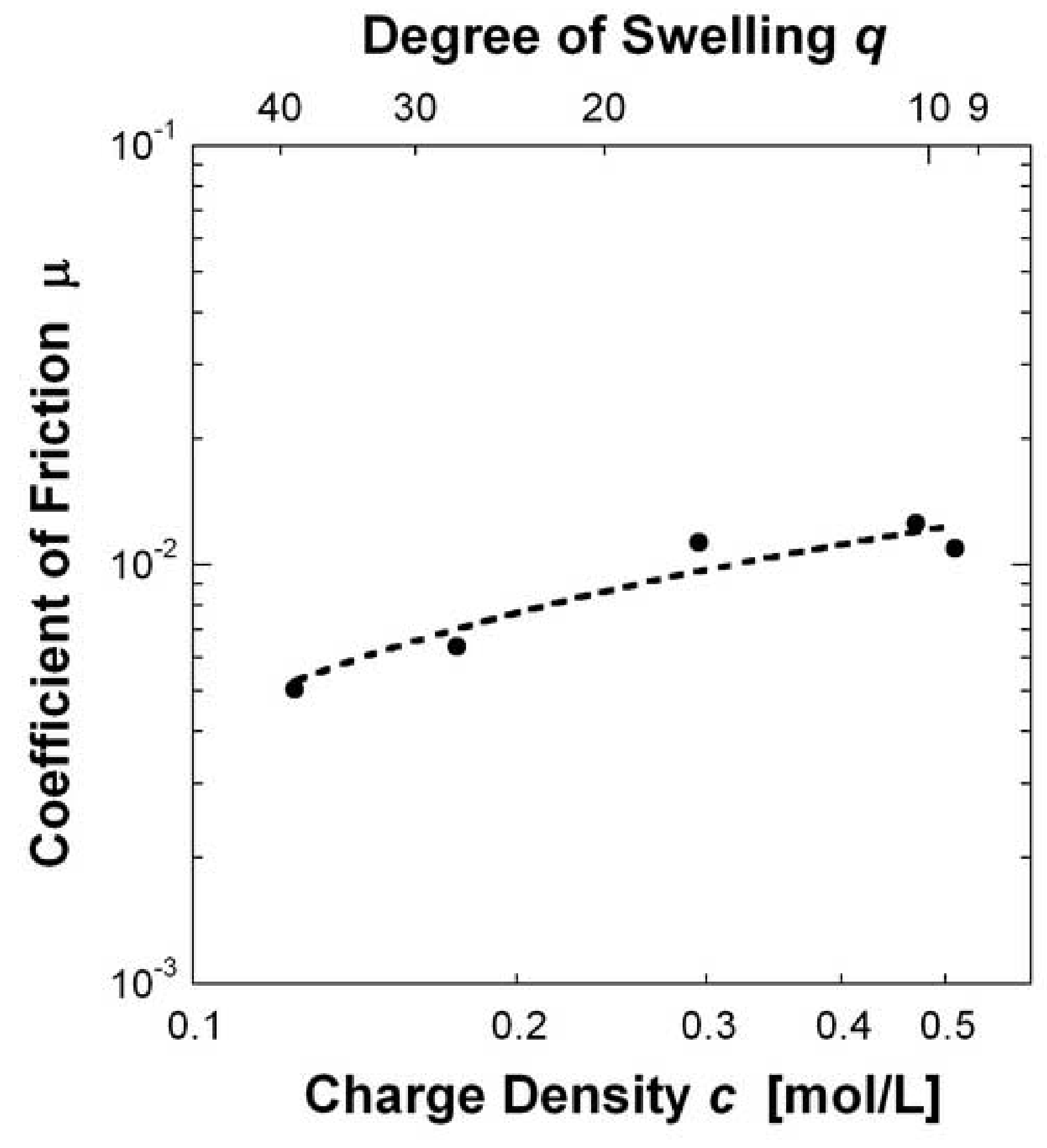

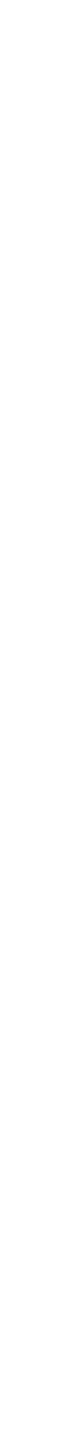

Degree of Swelling $q$ 
Figure $2 a$
Click here to download high resolution image

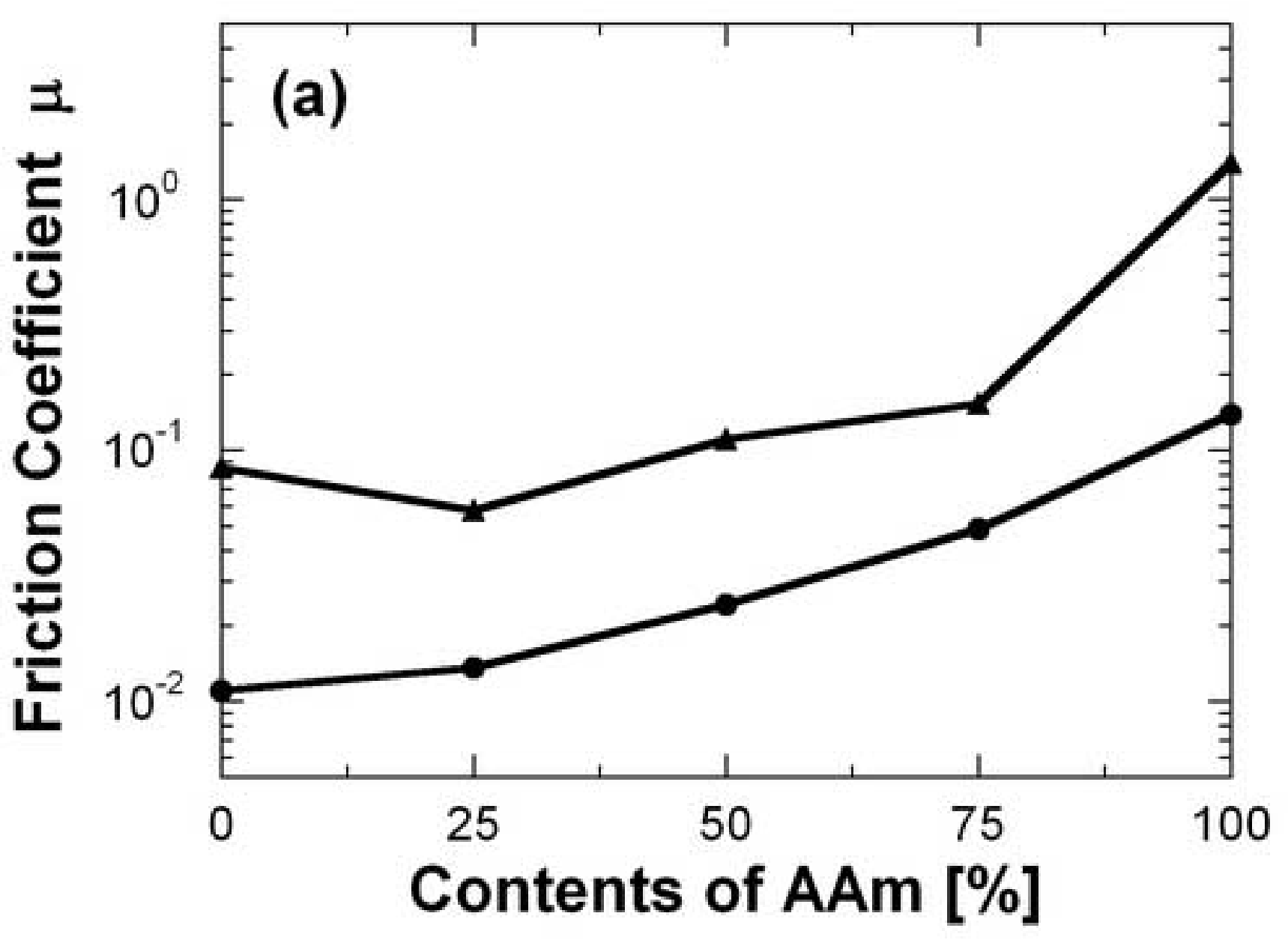


Click here to download high resolution image

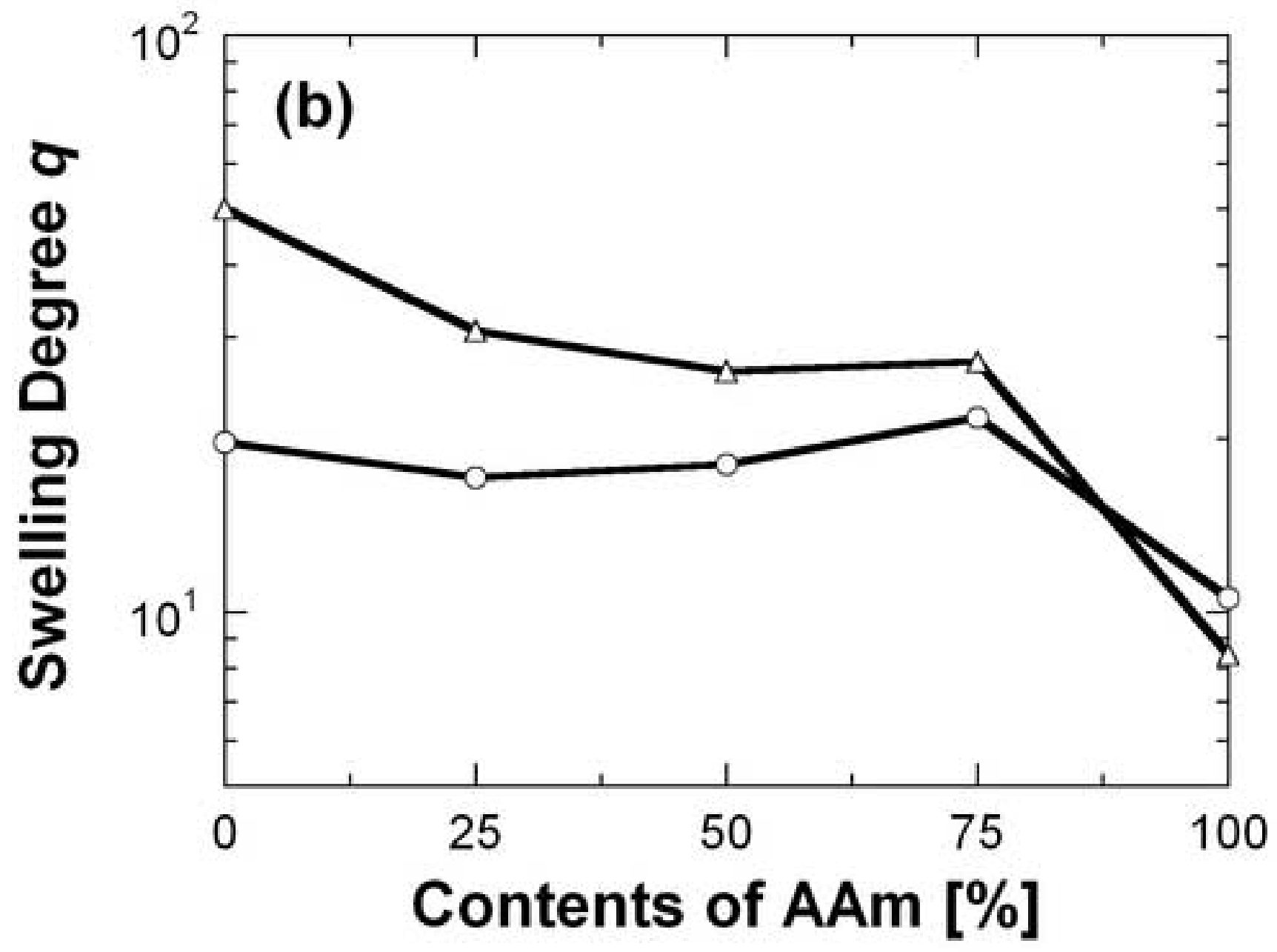


Figure 3

Click here to download high resolution image

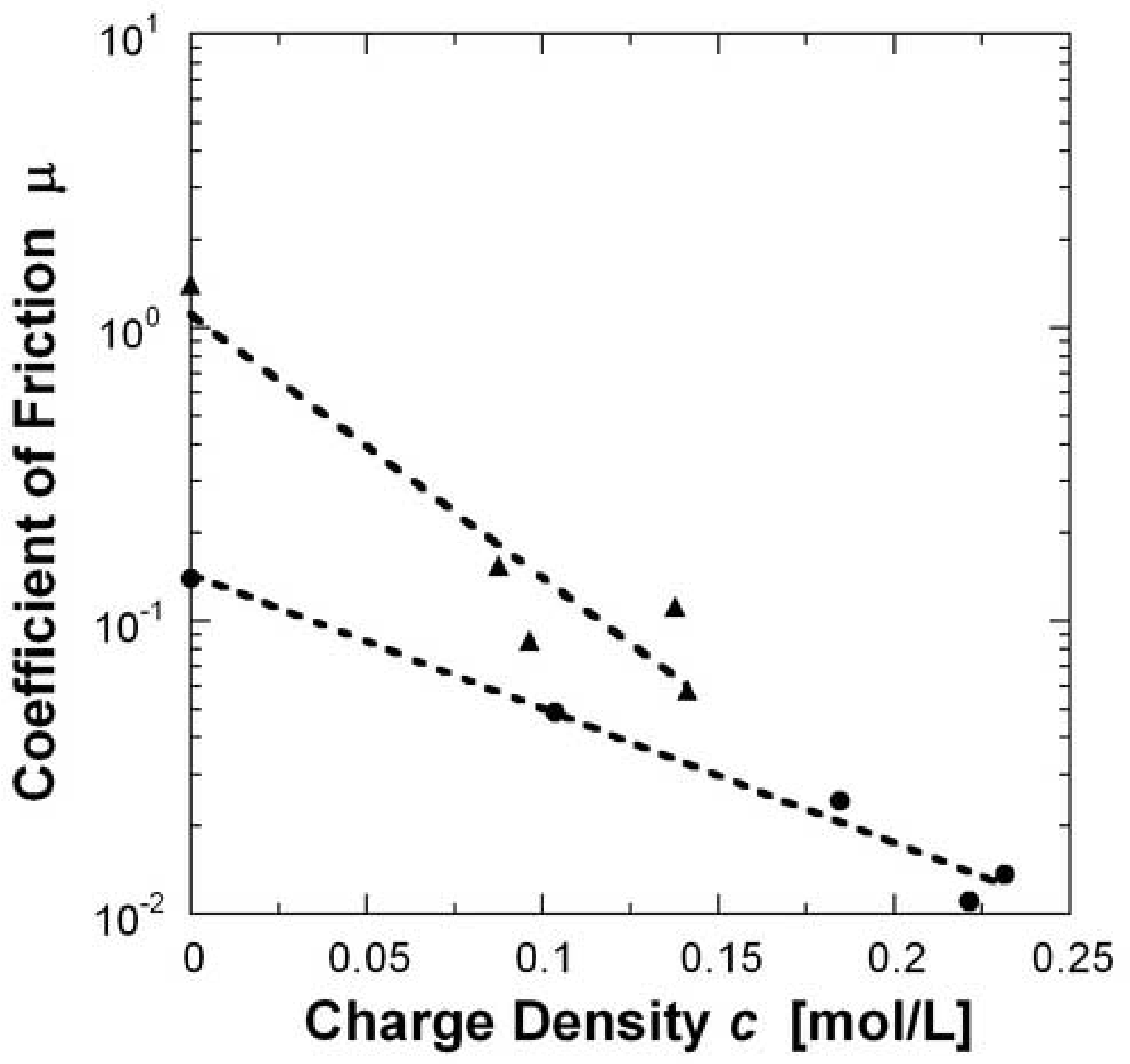


Figure $4 a$
Click here to download high resolution image

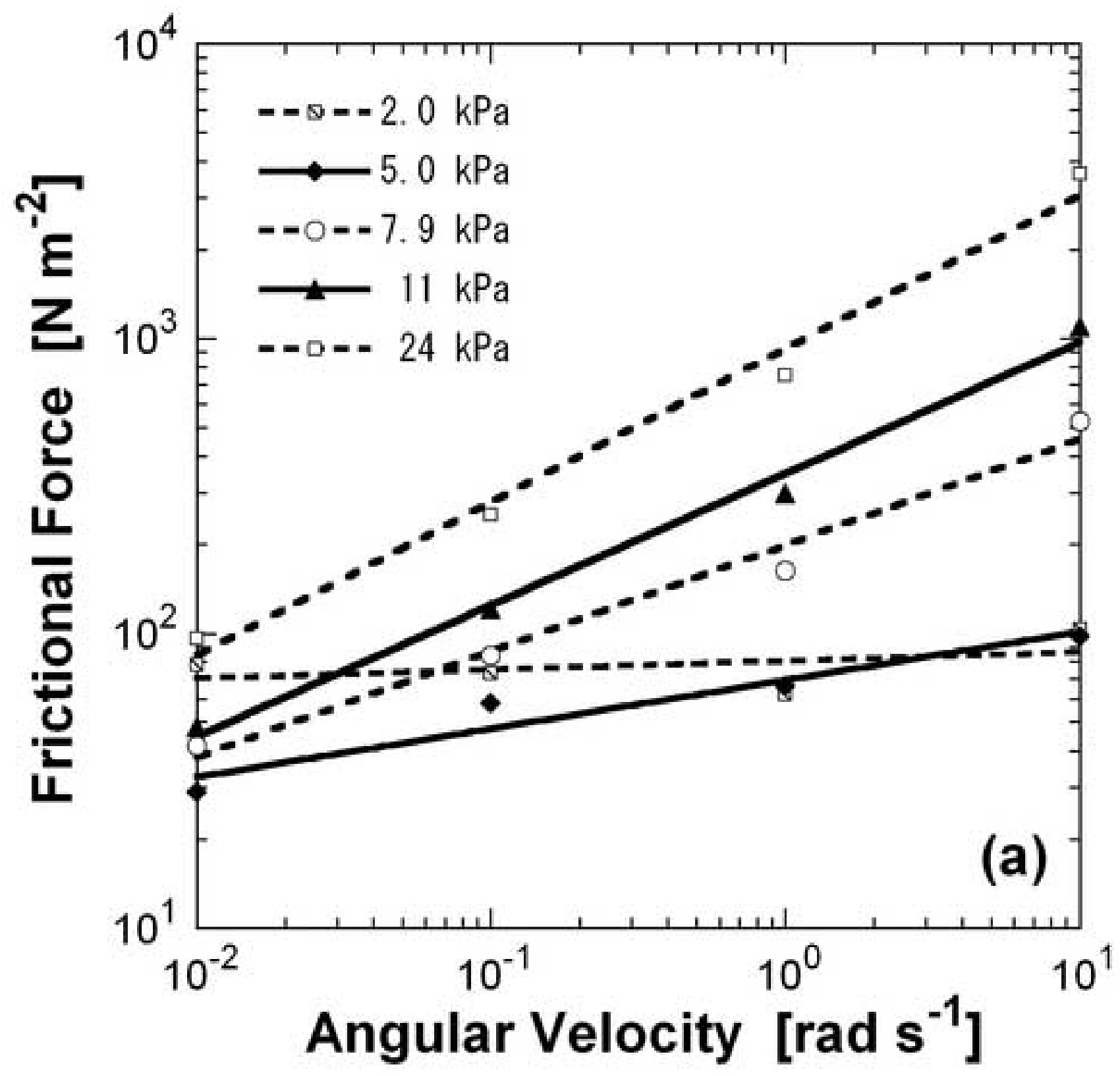


Figure $4 \mathrm{~b}$
Click here to download high resolution image

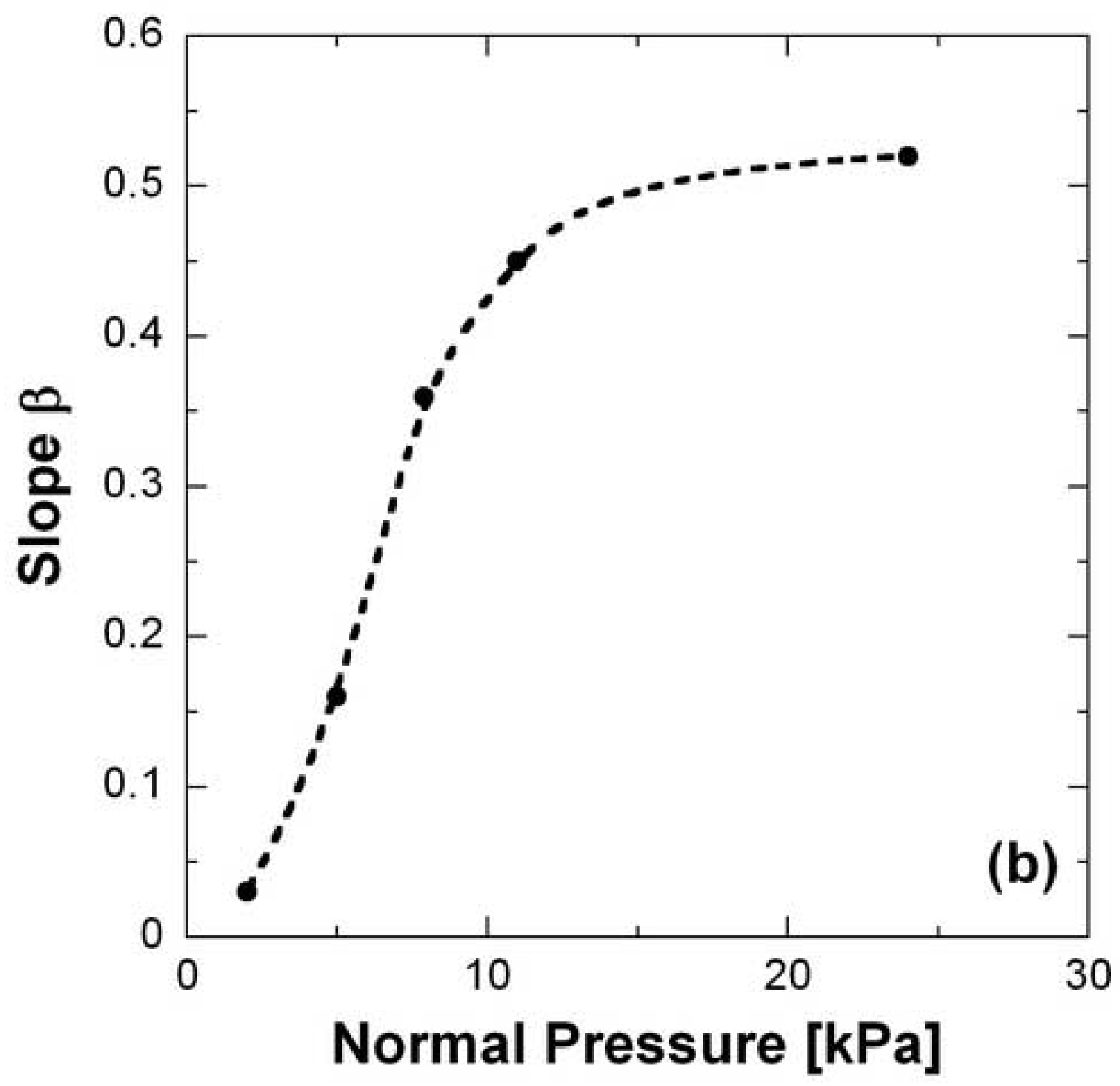


Figure 5
Click here to download high resolution image

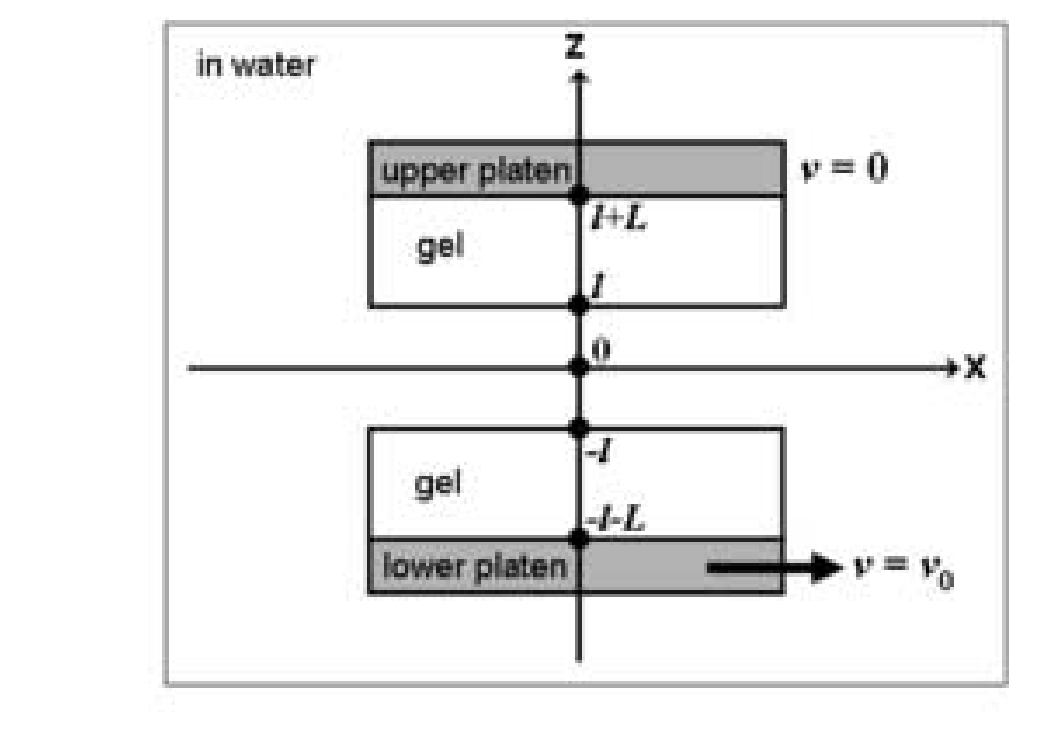

\section{e

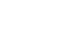


Figure 6

Click here to download high resolution image

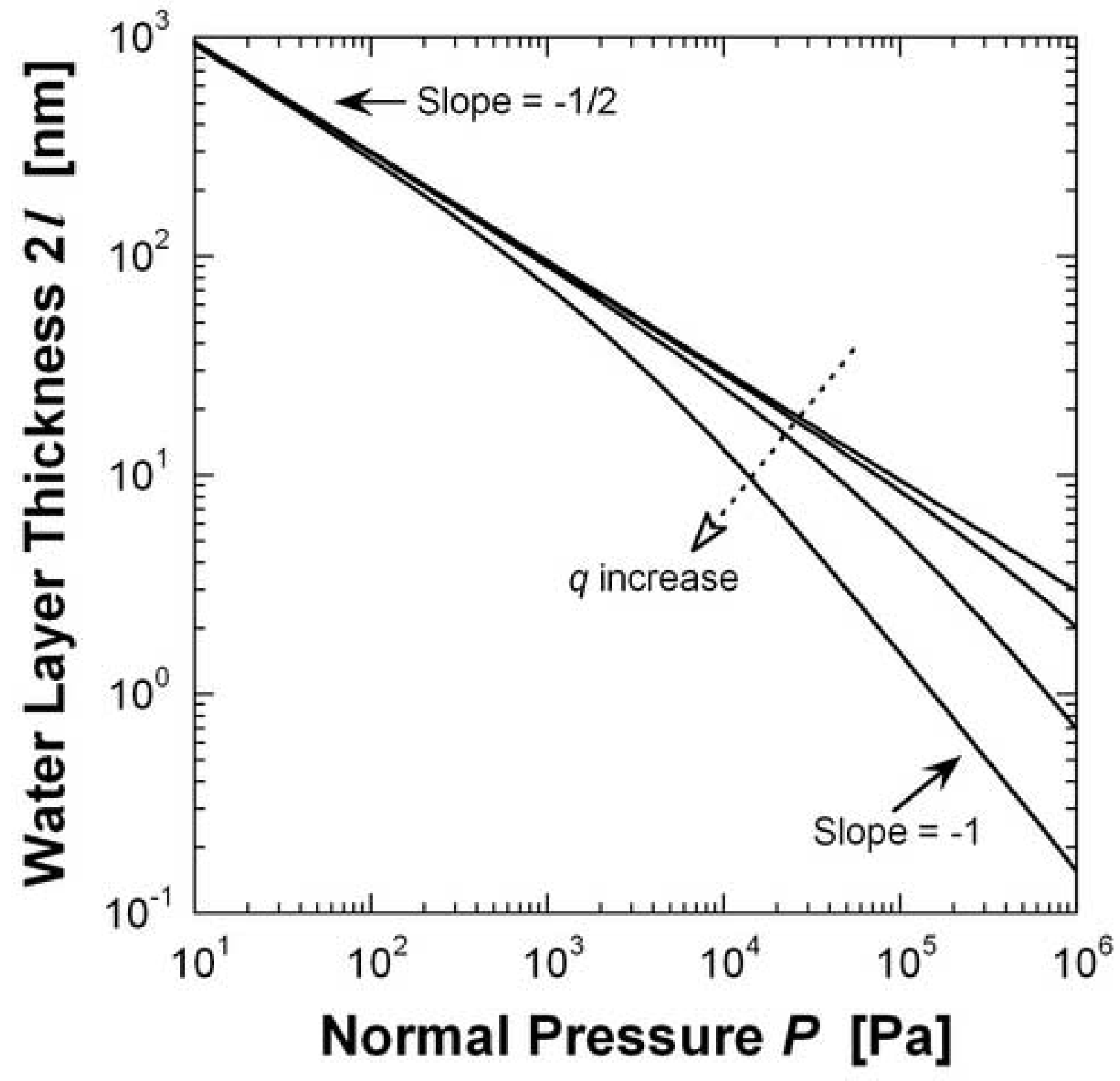


Figure 7
Click here to download high resolution image

\section{Charge Density c [mol/L]}

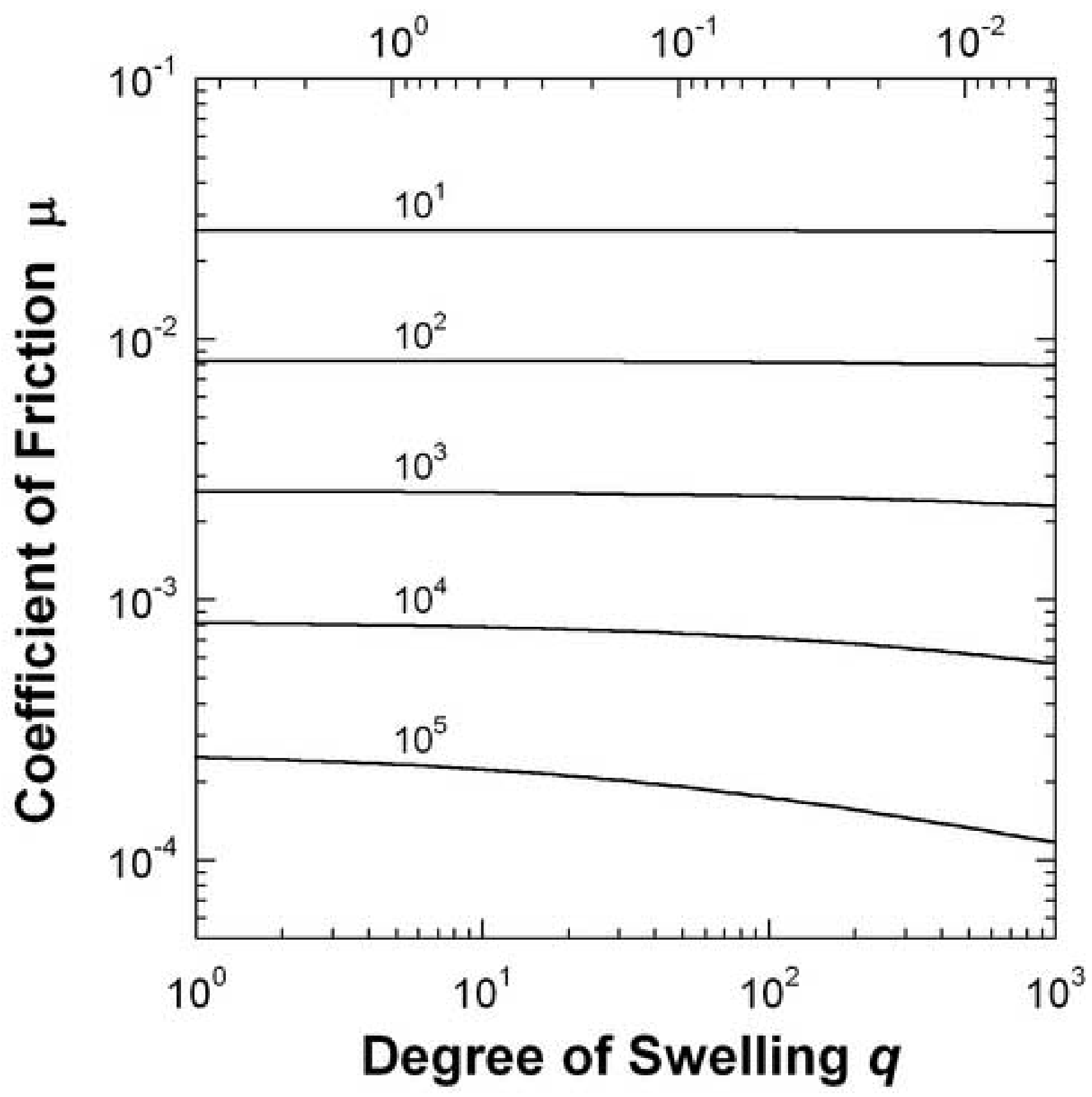


Figure 8
Click here to download high resolution image

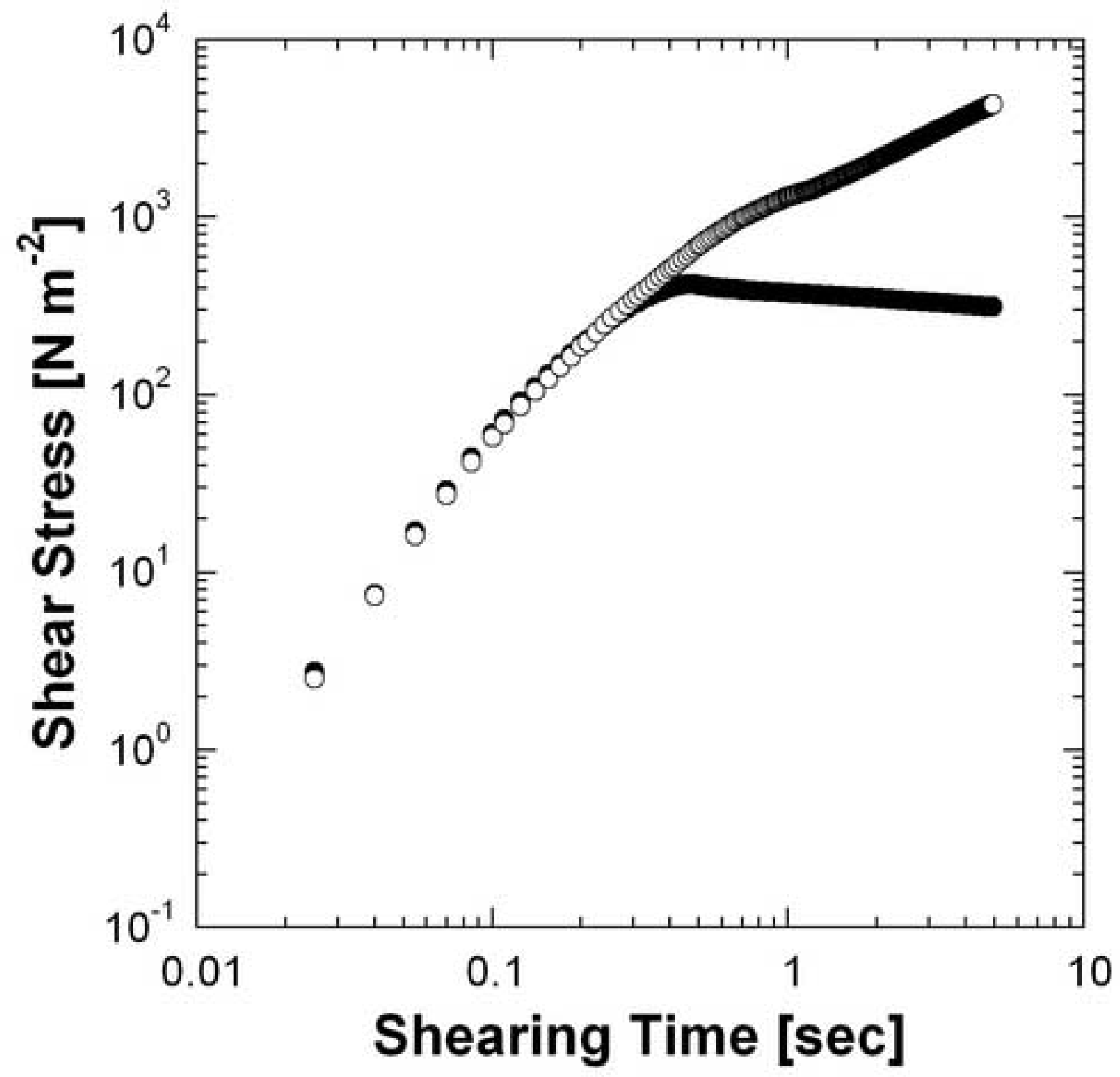

